\title{
A state space approach to periodic convolutional $\operatorname{codes}^{\star}$
}

\author{
Diego Napp ${ }^{1}$, Ricardo Pereira ${ }^{2}$, and Paula Rocha ${ }^{3}$ \\ ${ }^{1}$ CIDMA - Center for Research and Development in Mathematics and Applications, \\ Department of Mathematics, University of Aveiro, Aveiro, Portugal diego@ua.pt \\ 2 CIDMA - Center for Research and Development in Mathematics and Applications, \\ Department of Mathematics, University of Aveiro, Aveiro, Portugal \\ ricardopereira@ua.pt \\ ${ }^{3}$ SYSTEC, Faculty of Engineering, University of Porto, Portugal mprocha@fe.up.pt
}

\begin{abstract}
In this paper we study periodically time-varying convolutional codes by means of input-state-output representations. Using these representations we investigate under which conditions a given time-invariant convolutional code can be transformed into an equivalent periodic time-varying one. The relation between these two classes of convolutional codes is studied for period 2. We illustrate the ideas presented in this paper by constructing a periodic time-varying convolutional code from a time-invariant one. The resulting periodic code has larger free distance than any time-invariant convolutional code with equivalent parameters.
\end{abstract}

Keywords: Convolutional codes, Periodically time-varying codes, Input-stateoutput representations

\section{Introduction}

Convolutional codes [10] are an important type of error correcting codes that can be represented as a time-invariant discrete linear system over a finite field [20]. They are used to achieve reliable data transfer, for instance, in mobile communications, digital video and satellite communications [10,23].

Since the sixties it has been widely known that convolutional codes and linear systems defined over a finite field are essentially the same objects [20]. More recently, there has been a new and increased interest in this connection and many

\footnotetext{
* This work was supported in part by the Portuguese Foundation for Science and Technology (FCT-Fundação para a Ciência e a Tecnologia), through CIDMA - Center for Research and Development in Mathematics and Applications, within project UID/MAT/04106/2013 and also by Project POCI-01-0145-FEDER-006933 - SYSTEC - Research Center for Systems and Technologies - funded by FEDER funds through COMPETE2020 - Programa Operacional Competitividade e Internacionalização (POCI) - and by national funds through FCT - Fundação para a Ciência e a Tecnologia.
} 
advances have been derived from using the system theoretical framework when dealing with convolutional codes, see $[12,17]$. Most of the large body of literature on convolutional codes and on the relation of these codes with linear systems has been devoted to the "time-invariant" case.

In this work we aim at studying time-varying convolutional codes from a system theoretical point of view. These codes have attracted much attention after Costello conjectured in [5] that nonsystematic time-varying convolutional codes can attain can larger free distance than the nonsystematic time-invariant ones. Since then, several researchers have investigated such codes $[3,15,16,18]$. Moreover, in combination with wavelets [6] time-varying convolutional codes yield unique trellis structures that resulted in fast and low computational complexity decoding algorithms. However, little is known on the relation of time-varying convolutional codes and time-varying linear systems and very few general constructions of time-varying convolutional codes with designed distances are known.

Here we deal with periodically time-varying convolutional codes (for short, periodic codes) using input-state-output representations, and investigate some of their special properties and structures. In particular, we associate periodic codes with suitably defined time-invariant convolutional codes. This allows us to derive constructions of (periodic) input-state-output representations for periodic codes from the better understood time-invariant class.

\section{Preliminaries}

In the sequel we shall follow the system theory notation and consider column vectors rather than row vectors.

\subsection{Time-invariant convolutional codes}

A time-invariant convolutional code is a set of finite support sequences, called codewords, obtained as the image of a polynomial shift operator (the encoder) acting on finite support sequences that correspond to the original information. More precisely this can be defined as follows.

Definition 1. Let $\mathbb{F}$ be a finite field and $n, k$ be positive integers with $k<n$. A time-invariant convolutional code $\mathcal{C}$ of rate $k / n$ is a set of finite support sequences described as

$$
\mathcal{C}=\left\{v: v(\ell)=\left(G\left(\sigma^{-1}\right) u\right)(\ell) ; \ell \in \mathbb{N}_{0}, u \in\left[\left(\mathbb{F}^{k}\right)^{\mathbb{N}_{0}}\right]_{\mathrm{FS}}\right\}
$$

where $G(z) \in \mathbb{F}^{n \times k}[z]$ is a full column rank $n \times k$ polynomial matrix over $\mathbb{F}$, called the encoder, $u$ taking values in $\mathbb{F}^{k}$ is the information sequence and $v$ is the codeword. Moreover, $\sigma^{-1}$ denotes the shift $\left(\sigma^{-1} u\right)(\ell)=u(\ell-1)$, and the subindex FS affecting a set of sequences indicates that only its finite support elements are considered. 
The encoders of a code $\mathcal{C}$ are not unique; however they only differ by right multiplication by unimodular matrices over $\mathbb{F}[z]$. An encoder matrix $G$ is called basic if it has a polynomial right inverse; from now on we shall only consider basic encoders, and refer to them simply as encoders. The encoder $G$ is called minimal if the sum of its column degrees attains the minimal possible value.

We define the degree $\delta$ of a convolutional code as the sum of the column degrees of one, and hence any, minimal encoder. Note that the list of column degrees (also known as Forney indices) of a minimal encoder is unique up to a permutation. The maximum of the Forney indices is called the memory of a code, and is denoted by $m$. A code $\mathcal{C}$ of rate $k / n$, degree $\delta$ and memory $m$ is said to be an $(n, k, \delta)$ code or an $(n, k, \delta, m)$ code if the memory is to be specified.

\subsection{Periodically time-varying convolutional codes}

In this work we consider convolutional $\operatorname{codes} \mathcal{C}$ with $P$-periodic encoders, i.e.:

$$
\begin{array}{r}
\mathcal{C}=\left\{v: v(P \ell+t)=\left(G^{t}\left(\sigma^{-1}\right) u\right)(P \ell+t) ; t=0, \ldots, P-1 ;\right. \\
\left.\ell \in \mathbb{N}_{0}, u \in\left[\left(\mathbb{F}^{k}\right)^{\mathbb{N}_{0}}\right]_{F S}\right\},
\end{array}
$$

where each $G^{t}(z)$ is an $n \times k$ time-invariant (basic) encoder. Such codes will be called P-periodic.

Inspired by the ideas developed in [13] and [1] for the case of behaviors, considering the linear map

$$
L_{p}:\left(\mathbb{F}^{n}\right)^{\mathbb{N}_{0}} \rightarrow\left(\mathbb{F}^{P n}\right)^{\mathbb{N}_{0}}
$$

defined by

$$
\left(L_{p} v\right)(\ell)=\left[\begin{array}{c}
v(P \ell) \\
v(P \ell+1) \\
\vdots \\
v(P \ell+P-1)
\end{array}\right], P \in \mathbb{N}
$$

we associate with $\mathcal{C}$ a time-invariant convolutional code $\mathcal{C}^{L}$, the lifted version of $\mathcal{C}$, defined as

$$
\mathcal{C}^{L}=\left\{\widetilde{v} \in\left(\mathbb{F}^{P n}\right)^{\mathbb{N}_{0}}: \widetilde{v}=L_{p} v, v \in \mathcal{C}\right\} .
$$

Note that, since

$$
\left(G^{t}\left(\sigma^{-1}\right) u\right)(P \ell+t)=\left(\left(\sigma^{t} G^{t}\left(\sigma^{-1}\right)\right) u\right)(P \ell),
$$

the equation in (1) can also be written as

$$
\left(\Omega_{P, n}(\sigma) v\right)(P \ell)=\left(G\left(\sigma, \sigma^{-1}\right) u\right)(P \ell), \ell \in \mathbb{N}_{0},
$$


where for $r \in \mathbb{N}$

$$
\Omega_{P, r}(\sigma)=\left[\begin{array}{c}
I_{r} \\
\sigma I_{r} \\
\vdots \\
\sigma^{P-1} I_{r}
\end{array}\right]
$$

is a polynomial matrix operator in the shift $\sigma$ and

$$
G\left(\sigma, \sigma^{-1}\right)=\left[\begin{array}{c}
G^{0}\left(\sigma^{-1}\right) \\
\sigma G^{1}\left(\sigma^{-1}\right) \\
\vdots \\
\sigma^{P-1} G^{P-1}\left(\sigma^{-1}\right)
\end{array}\right]
$$

is a polynomial matrix operator in the shifts $\sigma$ and $\sigma^{-1}$.

Moreover, it is possible to show that the matrix $G$ can be decomposed as

$$
G\left(\sigma, \sigma^{-1}\right)=G^{L}\left(\sigma^{-P}\right) \Omega_{P, k}(\sigma)
$$

where

$$
G^{L}\left(\sigma^{-1}\right)=\left[G^{L_{0}}\left(\sigma^{-1}\right)\left|G^{L_{1}}\left(\sigma^{-1}\right)\right| \cdots \mid G^{L_{P-1}}\left(\sigma^{-1}\right)\right]
$$

and the blocks $G^{L_{j}}\left(\sigma^{-1}\right)$ have size $P n \times k, j=0, \ldots, P-1$.

Thus, the lifted code can be represented as

$$
\mathcal{C}^{L}=\left\{\widetilde{v}: \widetilde{v}(\ell)=\left(G^{L}\left(\sigma^{-1}\right) \widetilde{u}\right)(\ell), \ell \in \mathbb{N}_{0}, \widetilde{u} \in\left[\left(\mathbb{F}^{k P}\right)^{\mathbb{N}_{0}}\right]_{F S}\right\},
$$

where $\widetilde{v}=L_{P} v$ and $\widetilde{u}=L_{P} u$.

\subsection{Distance properties}

In recent years great effort has been dedicated to developing constructions of non-binary convolutional codes having good distance $[2,9,14]$. However, in contrast to block codes, the theoretical tools for the construction of convolutional codes with good designed distance have not been fully exploited. In fact, most convolutional codes used in practice have been found by systematic computer search and their distance properties must be also computed by full search.

One of our objectives will be the construction of convolutional codes with a large free distance, which is defined as follows.

Definition 2. The free distance of a convolutional code $\mathcal{C}$ is given by

$$
d_{\text {free }}(\mathcal{C})=\min \left\{\sum_{\ell=0}^{\infty} \operatorname{wt}(v(\ell)): v \in \mathcal{C} \backslash\{0\}\right\},
$$

where wt denotes the Hamming weight, that is, $\mathrm{wt}(v(\ell))$ corresponds to the number of nonzero components of $v(\ell)$. 
Rosenthal and Smarandache [21] showed that the free distance of a time-invariant $(n, k, \delta)$ convolutional code is upper bounded by

$$
d_{\text {free }}(\mathcal{C}) \leq(n-k)\left(\left\lfloor\frac{\delta}{k}\right\rfloor+1\right)+\delta+1 .
$$

This bound is called the generalized Singleton bound. It is well-known [21] that over sufficiently large finite fields, there always exist convolutional codes that achieve this bound for any given set of parameters $(n, k, \delta)$.

However, in this paper we will consider instead the Griesmer bound defined in the next theorem. This bound is always less than or equal to the generalized Singleton bound, and can be considerably lower for codes over small fields, whereas it coincides with the Singleton for codes over sufficiently large finite fields.

Theorem 1. $[7,10,19]$ Let $(n, k, \delta, m)$ be a 4-tuple of nonnegative integers such that $k<n$, consider $q \in \mathbb{N}$ and $\hat{\mathbb{N}}=\left\{\begin{array}{ll}\mathbb{N} & \text { if } k m=\delta \\ \mathbb{N}_{0} & \text { if } k m>\delta\end{array}\right.$. Let further

$$
G B_{q}(n, k, \delta, m)=\max \left\{d^{\prime}: \sum_{j=0}^{k(m+i)-\delta-1}\left\lceil\frac{d^{\prime}}{q^{j}}\right\rceil \leq n(m+i), \forall i \in \hat{\mathbb{N}}\right\} .
$$

Then, every $(n, k, \delta, m)$-convolutional code $\mathcal{C}$ over the field $\mathbb{F}_{q}$ is such that $d_{\text {free }}(\mathcal{C}) \leq G B_{q}(n, k, \delta, m)$.

$G B_{q}(n, k, \delta, m)$ is known as the Griesmer bound.

\section{$3 \quad$ State space realizations}

A state space system

$$
\left\{\begin{array}{c}
x(\ell+1)=A x(\ell)+B u(\ell) \\
v(\ell)=C x(\ell)+D u(\ell)
\end{array}, l \in \mathbb{N}_{0},\right.
$$

denoted by $(A, B, C, D)$, where $A \in \mathbb{F}^{\delta \times \delta}, B \in \mathbb{F}^{\delta \times k}, C \in \mathbb{F}^{n \times \delta}$ and $D \in \mathbb{F}^{n \times k}$, is said to be a state space realization of the time-invariant $(n, k, \delta)$ convolutional code $\mathcal{C}$ if $\mathcal{C}$ is the set of finite support output sequences $v$ corresponding to finite support input sequences $u$ and zero inicial conditions, i.e., $x(0)=0$.

Remark 1. This definition implicitly assumes that $(A, B, C, D)$ is a minimal realization of $\mathcal{C}$, i.e., that $A$ has the minimal possible dimension. This implies that $A$ is nilpotent, $(A, B)$ is controllable and $(A, C)$ is observable, i.e., the polynomial matrices $\left[z^{-1} I-A \mid B\right]$ and $\left[\begin{array}{c}z^{-1} I-A \\ C\end{array}\right]$ have, respectively, right and left polynomial inverses (in $z^{-1}$ ). 
State space realizations for convolutional codes can be obtained as minimal state space realizations of minimal encoders.

The next proposition, adapted from [8, Proposition 2.3], provides a state space realization for a given (not necessarily minimal) encoder.

Proposition 1. Let $G \in \mathbb{F}^{n \times k}[z]$ be a polynomial matrix with rank $k$ and column degrees $\nu_{1}, \ldots, \nu_{k}$. Consider $\bar{\delta}=\sum_{i=1}^{k} \nu_{i}$. Let $G$ have columns $g_{i}=\sum_{\ell=0}^{\nu_{i}} g_{\ell, i} z^{\ell}$, $i=1, \ldots, k$ where $g_{\ell, i} \in \mathbb{F}^{n}$. For $i=1, \ldots, k$ define the matrices

$$
A_{i}=\left[\begin{array}{rrrr}
0 & \cdots & \cdots & 0 \\
1 & & & \vdots \\
\ddots & & \vdots \\
& & 1 & 0
\end{array}\right] \in \mathbb{F}^{\nu_{i} \times \nu_{i}}, B_{i}=\left[\begin{array}{c}
1 \\
0 \\
\vdots \\
0
\end{array}\right] \in \mathbb{F}^{\nu_{i}}, C_{i}=\left[g_{1, i} \cdots g_{\nu_{i}, i}\right] \in \mathbb{F}^{n \times \nu_{i}}
$$

Then a state space realization of $G$ is given by the matrix quadruple $(A, B, C, D) \in$ $\mathbb{F}^{\bar{\delta} \times \bar{\delta}} \times \mathbb{F}^{\bar{\delta} \times k} \times \mathbb{F}^{n \times \bar{\delta}} \times \mathbb{F}^{n \times k}$ where

$A=\left[\begin{array}{llll}A_{1} & & \\ & \ddots & \\ & & A_{k}\end{array}\right], B=\left[\begin{array}{llll}B_{1} & & \\ & \ddots & \\ & & B_{k}\end{array}\right], C=\left[C_{1} \cdots C_{k}\right], D=\left[g_{0,1} \cdots g_{0, k}\right]=G(0)$.

In the case where $\nu_{i}=0$ the ith block is missing and in $B$ a zero column occurs.

In this realization $(A, B)$ is controllable, and if $G$ is a minimal encoder, $(A, C)$ is observable.

\section{Constructing periodically time-varying convolutional codes}

In comparison to the literature on time-invariant convolutional codes, there exist few algebraic constructions of time-varying convolutional codes with good properties $[11,22]$. Here we present a new technique to build time-varying convolutional codes from time-invariant ones. In particular, in this section we focus on constructing 2-periodic codes with optimal free distance. We illustrate our approach by means of an example of a 2-periodic $(3,2,2,1)$ code having larger distance than any $(3,2,2,1)$ time-invariant convolutional code.

We first investigate the problem of finding periodic state space representations of periodic convolutional codes. As shown in Section 2.2, using a lifting technique one can transform a time-varying periodic linear system into an equivalent timeinvariant one. Following [1], we study the relationship between the periodic state space representations of a given code and the time-invariant state space representations of its lifted version. For the sake of simplicity we assume that the period 
is $P=2$. However, whereas in [1] only single-input/single-output systems were considered, here we deal with codes of general rate $k / n$ that are closely related to multi-input/multi-output (MIMO) systems.

Assume that $\Sigma(\cdot)=(A(\cdot), B(\cdot), C(\cdot), D(\cdot))$ is a $\delta$-dimensional state space representation of a code $\mathcal{C}$, as present below:

$$
\left\{\begin{array}{c}
x(\ell+1)=A(\ell) x(\ell)+B(\ell) u(\ell) \\
v(\ell)=C(\ell) x(\ell)+D(\ell) u(\ell)
\end{array}, l \in \mathbb{N}_{0}\right.
$$

where $(A(\cdot), B(\cdot), C(\cdot), D(\cdot)) \in \mathbb{F}^{\delta \times \delta} \times \mathbb{F}^{\delta \times k} \times \mathbb{F}^{n \times \delta} \times \mathbb{F}^{n \times k}$ are periodic functions with period 2. Letting

$$
\begin{aligned}
w(\ell) & =x(2 \ell) \\
u^{L}(\ell) & =\left[\begin{array}{c}
u(2 \ell) \\
u(2 \ell+1)
\end{array}\right] \\
v^{L}(\ell) & =\left[\begin{array}{c}
v(2 \ell) \\
v(2 \ell+1)
\end{array}\right]
\end{aligned}
$$

we obtain the following time-invariant $\delta$-dimensional state space representation $\Sigma=(E, F, H, J)$ for the lifted code $\mathcal{C}^{L}$ :

$$
\left\{\begin{array}{c}
w(\ell+1)=E w(\ell)+F u^{L}(\ell) \\
v^{L}(\ell)=H z(\ell)+J u^{L}(\ell)
\end{array},\right.
$$

with

$$
\begin{array}{ll}
E=A(1) A(0) & F=\left[\begin{array}{cc}
A(1) B(0) & B(1)
\end{array}\right] \\
H=\left[\begin{array}{c}
C(0) \\
C(1) A(0)
\end{array}\right] \quad J=\left[\begin{array}{cc}
D(0) & 0 \\
C(1) B(0) & D(1)
\end{array}\right] .
\end{array}
$$

The representation $\Sigma=(E, F, H, J)$ of $\mathcal{C}^{L}$ is said to be induced by the representation $\Sigma(\cdot)=(A(\cdot), B(\cdot), C(\cdot), D(\cdot))$ of $\mathcal{C}$, or equivalently, $\Sigma(\cdot)$ is said to induce $\Sigma^{L}$. Moreover, a time-invariant representation $\Sigma=(E, F, H, J)$ of $\mathcal{C}^{L}$ is called induced whenever it is induced by some periodic representation $\Sigma(\cdot)$ of $\mathcal{C}$.

The following proposition is a generalization of [1, Proposition 3.1] (with identical proof) and characterizes induced representations.

Proposition 2. Let $\mathcal{C}$ be a 2-periodic code and $\mathcal{C}^{L}$ the lifted code associated to $\mathcal{C}$. Then a $\delta$-dimensional state space representation $\Sigma=(E, F, H, J)$ of $\mathcal{C}^{L}$, with

$$
\begin{array}{ll}
E \in \mathbb{F}^{\delta \times \delta} & F=\left[\begin{array}{ll}
F_{1} & F_{2}
\end{array}\right] \in \mathbb{F}^{\delta \times 2 k} \\
H=\left[\begin{array}{l}
H_{1} \\
H_{2}
\end{array}\right] \in \mathbb{F}^{2 n \times \delta} & J=\left[\begin{array}{ll}
J_{11} & J_{12} \\
J_{21} & J_{22}
\end{array}\right] \in \mathbb{F}^{2 n \times 2 k} .
\end{array}
$$


is induced if and only if

$$
\operatorname{rank} \mathcal{M}=\left[\begin{array}{cc}
E & F_{1} \\
H_{2} & J_{21}
\end{array}\right] \leq \delta
$$

Moreover, in this case, decomposing the matrix $\mathcal{M}$ as

$$
\mathcal{M}=\left[\begin{array}{l}
N_{1} \\
N_{2}
\end{array}\right]\left[\begin{array}{ll}
Q_{1} & Q_{2}
\end{array}\right]
$$

the 2-periodic $\delta$-dimensional state space representation of $\mathcal{C}$ that induces $\Sigma^{L}$ is $\Sigma(\cdot)=(A(\cdot), B(\cdot), C(\cdot), D(\cdot))$, where

$$
\begin{array}{llll}
A(0)=Q_{1} & A(1)=N_{1} & B(0)=Q_{2} & B(1)=F_{2} \\
C(0)=H_{1} & C(1)=N_{2} & D(0)=J_{11} & D(1)=J_{22} .
\end{array}
$$

Hence, Proposition 2 characterizes the state space realizations of time-invariant convolutional codes from which (2-periodic) time-varying codes can be constructed. In this way one can use the large body of literature and constructions for the time-invariant case in order to build time-varying convolutional codes with good properties. In the next section we illustrate this with an example.

\subsection{2-periodic $(3,2,2,1)$ convolutional code with free distance 4}

It is know from the literature that $(3,2,2,1)$ time-invariant convolutional codes have at most free distance 3 , whereas the Griesmer bound for this kind of codes is 4 . In this section we construct a 2-periodic $(3,2,2,1)$ convolutional code with free distance 4 based on the construction of a time-invariant code whose state space realization is induced by a 2 -periodic realization. This shows that timevarying convolutional codes can attain larger free distance than time-invariant ones.

Example 1. Consider the $(6,4,2,1)$ time-invariant convolutional code, $\mathcal{C}^{L}$, over $\mathbb{F}_{2}$ with generator matrix $G=G_{1} z+G_{0}$, where

$$
G_{0}=\left[\begin{array}{llll}
1 & 1 & 0 & 0 \\
0 & 1 & 0 & 0 \\
1 & 0 & 0 & 0 \\
1 & 0 & 1 & 0 \\
1 & 1 & 1 & 1 \\
0 & 1 & 1 & 0
\end{array}\right] \quad \text { and } \quad G_{1}=\left[\begin{array}{llll}
0 & 0 & 1 & 1 \\
0 & 0 & 0 & 1 \\
0 & 0 & 0 & 1 \\
0 & 0 & 0 & 0 \\
0 & 0 & 0 & 0 \\
0 & 0 & 0 & 0
\end{array}\right] .
$$

As $\left[\begin{array}{l}G_{0} \\ G_{1}\end{array}\right]$ is an encoder of a $(12,3)$ block code of free distance 4 , we conclude that the free distance of $\mathcal{C}^{L}$ is at most 4 . Moreover, it can be computed via a program that the free distance of $\mathcal{C}^{L}$ is indeed 4. Since the column degrees of $G$ 
are $\nu_{1}=0, \nu_{2}=0, \nu_{3}=1, \nu_{4}=1$, by Proposition 1 , a state space realization of $G$ is given by $(E, F, H, J) \in \mathbb{F}^{2 \times 2} \times \mathbb{F}^{2 \times 4} \times \mathbb{F}^{6 \times 2} \times \mathbb{F}^{6 \times 4}$ where

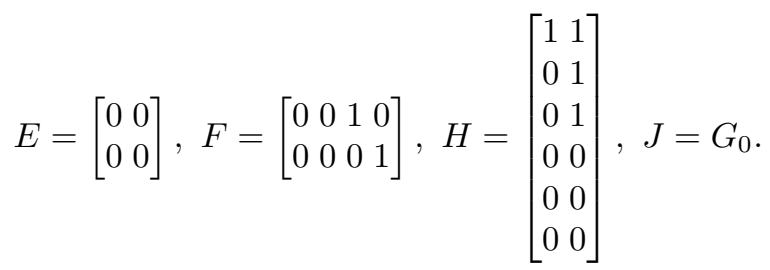

Now, the matrix $\mathcal{M}$ defined in Proposition 2 is:

$$
\mathcal{M}=\left[\begin{array}{llll}
0 & 0 & 0 & 0 \\
0 & 0 & 0 & 0 \\
0 & 0 & 1 & 0 \\
0 & 0 & 1 & 1 \\
0 & 0 & 0 & 1
\end{array}\right] .
$$

Since $\operatorname{rank} \mathcal{M}=2 \leq \delta$, by this proposition we conclude that realization (4) is induced by a 2 -periodic $(3,2,2,1)$ convolutional code, with realization $\Sigma(\cdot)=$ $(A(\cdot), B(\cdot), C(\cdot), D(\cdot))$ where

$$
\begin{aligned}
& A(0)=\left[\begin{array}{ll}
0 & 0 \\
0 & 0
\end{array}\right] \quad A(1)=\left[\begin{array}{ll}
0 & 0 \\
0 & 0
\end{array}\right] \quad B(0)=\left[\begin{array}{ll}
1 & 0 \\
0 & 1
\end{array}\right] \quad B(1)=\left[\begin{array}{ll}
1 & 0 \\
0 & 1
\end{array}\right] \\
& C(0)=\left[\begin{array}{ll}
1 & 1 \\
0 & 1 \\
0 & 1
\end{array}\right] \quad C(1)=\left[\begin{array}{ll}
1 & 0 \\
1 & 1 \\
0 & 1
\end{array}\right] \quad D(0)=\left[\begin{array}{ll}
1 & 1 \\
0 & 1 \\
1 & 0
\end{array}\right] \quad D(1)=\left[\begin{array}{ll}
1 & 0 \\
1 & 1 \\
1 & 0
\end{array}\right] \text {. }
\end{aligned}
$$

This 2-periodic code can also be described as in (1) with $P=2$, and $G^{t}(z)=$ $C(t)\left(z^{-1} I-A(t)\right)^{-1} B(t)+D(t)$, for $t=0,1$, which yields

$$
G^{0}(z)=\left[\begin{array}{ll}
1 & 1 \\
0 & 1 \\
0 & 1
\end{array}\right] z+\left[\begin{array}{ll}
1 & 1 \\
0 & 1 \\
1 & 0
\end{array}\right] \quad \text { and } \quad G^{1}(z)=\left[\begin{array}{ll}
1 & 0 \\
1 & 1 \\
0 & 1
\end{array}\right] z+\left[\begin{array}{ll}
1 & 0 \\
1 & 1 \\
1 & 0
\end{array}\right] .
$$

This example is equivalent to the one presented by Palazzo in [18].

\section{Conclusions}

In this paper we have studied the relation between time-invariant and timevarying convolutional codes by means of input-state-output representations. Using a well known lifting technique we have shown how it is possible to transform a given periodically time-varying convolutional code into a time-invariant one. Moreover, we have provided conditions, in terms of input-state-output representations, to transform a time-invariant convolutional code into a time-varying one. Using these ideas, we have illustrated how to construct a 2-periodic $(3,2,2,1)$ 
convolutional code with optimal free distance from a $(3,2,2,1)$ time-invariant one. This showed that time-varying convolutional codes can attain larger free distance than time-invariant ones. Constructions of periodic convolutional codes of higher periods and with other parameters are currently under investigation.

\section{References}

1. J.C. Aleixo, P. Rocha and J.C. Willems, State space representation of SISO periodic behaviors, Proceedings of the 50th IEEE Conference on Decision and Control and European Control Conference (CDC-ECC), Orlando, FL, USA, 12-15 Dec. 2011, pp. 1545-1550.

2. P. Almeida, D. Napp R. and R. Pinto, A new class of superregular matrices and MDP convolutional codes, Linear Algebra and its Applications, Vol. 439, No. 7, pp. 2145-2147, 2013.

3. I. Bocharova and B. Kudryashov, Rational rate punctured convolutional codes for soft-decision Viterbi decoding. IEEE Transactions on Information Theory Vol. 43, No. 4, pp. 1305-1313, 1997.

4. J-J Climent, V. Herranz, C. Perea and V. Tomás, A systems theory approach to periodically time-varying convolutional codes by means of their invariant equivalent, Applied Algebra, Algebraic Algorithms and Error-Correcting Codes M. BrasAmorós and T. Høholdt, eds, Springer Berlin Heidelberg, Vol. 5527, pp. 73-82, 2009.

5. D. Costello, Free distance bounds for convolutional codes, IEEE Transactions on Information Theory, Vol. 20, No. 3, pp. 356-365, 1974.

6. F. Fekri, M. Sartipi, R.M. Mersereau and R.W. Schafer, Convolutional codes using finite-field wavelets: time-varying codes and more, IEEE Transactions on Signal Processing, Vol. 53, No. 5, pp. 1881-1896, 2005.

7. H. Gluesing-Luerssen and W. Schmale, Distance bounds for convolutional codes and some optimal codes, arXiv:math/0305135v1, 2003.

8. H. Gluesing-Luerssen and G. Schneider, State space realizations and monomial equivalence for convolutional codes, Linear Algebra and its Applications, Vol. 425, pp. 518-533, 2007.

9. H. Gluesing-Luerssen, J. Rosenthal and R. Smarandache, Strongly-MDS convolutional codes, IEEE Transactions on Information Theory, Vol. 52, No. 2, pp. 584-598, 2006.

10. R. Johannesson and K.S. Zigangirov, Fundamentals of convolutional coding. IEEE press, New York, 1999.

11. J. Justesen, New convolutional code constructions and a class of asymptotically good time-varying codes, IEEE Transactions on Information Theory, Vol. 19, No. 2, pp. 220-225, 1973.

12. M. Kuijper and J.W. Polderman, Reed-Solomon list decoding from a systemtheoretic perspective, IEEE Transactions on Information Theory, Vol. 50, No. 2, pp. 259-571, 2004.

13. M. Kuijper and J. C. Willems, A behavioral framework for periodically timevarying systems, Proceedings of the 36th IEEE Conference on Decision $\mathcal{G}$ Control - CDC'97, Vol. 3, San Diego, California USA, 10-12 Dec. 1997, pp. 2013-2016.

14. G. La Guardia, Convolutional codes: techniques of construction, Computational and Applied Mathematics, Vol. 35, No. 2, pp. 501-517, 2016. 
15. P.J. Lee, There are many good periodically time-varying convolutional codes, IEEE Transactions on Information Theory, Vol. 35, No. 2, pp. 460-463, 1989.

16. M. Mooser, Some periodic convolutional codes better than any fixed code, IEEE Transactions on Information Theory, Vol. 29, No 5, pp. 750-751, 1983.

17. D. Napp, C. Perea and R. Pinto, Input-state-output representations and constructions of finite support 2D convolutional codes, Advances in Mathematics of Communications, Vol. 4, No. 4 pp. 533-545, 2010.

18. R. Palazzo, A time-varying convolutional encoder better than the best timeinvariant encoder, IEEE Transactions on Information Theory, Vol. 39, No. 3, pp. 1109-1110, 1993.

19. J.M. Porras and J.I. Curto, Classification of convolutional codes, Linear Algebra and its Applications, Vol. 432, No. 10, pp. 2701-2725, 2010.

20. J. Rosenthal, Connections between linear systems and convolutional codes, Codes, Systems, and Graphical Models, B. Marcus and J. Rosenthal, eds, Springer New York, Vol. 123, pp. 39-66, 2001.

21. J. Rosenthal and R. Smarandache, Maximum distance separable convolutional codes, Applicable Algebra in Engineering, Communication and Computing, Vol. 10, pp. 15-32, 1999.

22. D. Truhachev, K. Zigangirov and D. Costello, Distance bounds for periodically time-varying and tail-biting LDPC convolutional codes, IEEE Transactions on Information Theory, Vol. 56, No. 9, pp. 4301-4308, 2010.

23. A.J. Viterbi, Convolutional codes and their performance in communication systems, IEEE Transactions on Communication Technology, Vol. 19, No 5, pp. 751$772,1971$. 